\title{
DEP1 gene in wheat species with normal, compactoid and compact spikes
}

\author{
Valeriya Vavilova*, Irina Konopatskaia, Anastasia E. Kuznetsova, Alexandr Blinov and Nikolay P. Goncharov \\ From Belyaev Conference \\ Novosibirsk, Russia. 07-10 August 2017
}

\begin{abstract}
Background: In rice, a variant of DEP1 gene results in erect panicle architecture, well-developed vascular bundles, an increase in the number of grains per panicle and a consequent increase in the grain yield. Interestingly, DEP1 homologs are present in the other cereals including species of wheat and barley (Hordeum vulgare), even though they do not produce panicles but spikes. In barley, HvDEP1 alleles do not differ between strains of various ear types and geographic origins, while in at least three OSDEP1 variants have been described.

Results: In this work, we have studied the DEP1 gene from eight accessions which belong to four wheat species, T. monococcum, T. durum, T. compactum, and T. spelta, with either compact, compactoid or normal spike phenotypes. The nucleotide sequences of the 5th exon of DEP1 were determined for all eight accessions. Obtained sequences were species specific. Despite the interspecies diversity, all wheat sequences encoded polypeptides of the same size, similarly to the 5th exons of the DEP1 homologs in T. aestivum, T. urartu, and H. vulgare. For further study, the full-length sequences of the DEP1 gene for all four species were studied. The full-length DEP1 genomic copies were isolated from the genomic sequences of T. aestivum, T. urartu, and Aegilops tauschii.

The genome of tetraploid wheat T. durum contains two variants of the DEP1 originating from A and B genomes. In the hexaploid wheats T. aestivum, T. compactum, and T. spelta, three variants of this gene originating from A, B, and $D$ genomes were detected. DEP1 genes of the diploid wheats T. monococcum and T. urartu differ. It seems that a precursor of the DEP1 gene in T. monococcum originates from the wild progenitor T. boeoticum.
\end{abstract}

Conclusion: No DEP1-related differences of nucleotide sequences between the compact (or compactoid) and normal spike phenotypes in the tested wheat species were detected. Therefore, DEP1 gene does not directly participate in the control of the spike architecture in wheats.

\section{Background}

Spike shape is a taxonomically important characteristic of the genus Triticum L. (tribe Triticeae Dum., family Poaceae Barn.). Three main variants of spike shape, namely, normal, spelt and compact, are widely distributed among wheats. Cultivated wheat species possess relatively short square headed parallel-sided spikes - so-called "normal" spikes [1], while the spikes of Triticum spelta L., T. dicoccum (Schrank) Schuebl., and T. dicoccoides (Körn. ex Aschers. et Graebn.) Schweinf. are of pyramidal shape with elongated rachis and tenacious glumes ("spelt type") [1]. The third variant of spike morphology is called "compact";

\footnotetext{
* Correspondence: valeriya-vavilova@bionet.nsc.ru

Institute of Cytology and Genetics SB RAS, Novosibirsk, Russia
}

these are short, dense spikes with fewer spikelets per spike recently described in diploid $T$. sinskajae and two hexaploid species, T. compactum Host and T. sphaerococcum Perciv. [1]. Additionally, a subvariant "compactoid", which is characterized by less dense spikes as compared to "compact" type, is found in the hybrids of $T$. dicoccoides and $T$. dicoccum $[2,3]$.

Genetic control of the spike shape trait in wheats is poorly investigated. Several genes and loci related to spike shape trait have been identified and include the following: $Q, C, C 2, s c 1$ and $s c 2$ (for review: [4]). Among listed above $Q$ gene was localized on the chromosomes of homeological group 5 and molecularly cloned. Gene $C$ was located on the long arm of chromosome 2D, while 
the sequences and genome localization of the $C 2, s c 1$ and $s c 2$ remain unknown [3, 5-9]. The transcription factor gene, $Q$, has a pleiotropic effect on spike shape in polyploid wheat species [9]. The dominant $5 A Q$ and recessive $5 A q$ alleles differ in two nucleotide substitutions and determine either normal or spelt spike phenotype [9-11]. $5 D q$ and $5 B q$ alleles are involved in the suppression of the spelt phenotype [10]. No investigation supports the influence of $Q$ gene on the formation of compact and compactoid spikes of polyploid wheat species.

DENSE AND ERECT PANICLE 1 (DEP1) gene extensively investigated in rice could contribute to the spike shape trait in wheat (for review [12]). In rice the DEP1 gene located on the chromosome 9 pleiotropically controls several traits including panicle density, grain number per panicle and erect panicle among others. Experiments with near-isogenic lines showed that OsDEP1 gene is a dominant negative regulator of panicle architecture and grain number. The DEP1 gene encodes the phosphatidylethanolaminebinding (PEBP) protein consisting of the following modules: plant-specific $\mathrm{G}$ s subunit protein domain, three von Willebrand factor type $\mathrm{C}$ (VWFC) domains and a tumor necrosis factor receptor (TNFR)/nerve growth factor receptor (NGFR) family cysteine-rich domain [13]. The mutant DEP1 allele (dep1) possess a replacement of a $637 \mathrm{bp}$ stretch from the middle of the 5th exon by a short sequence $12 \mathrm{bp}$ in length which results in loss of cystein-rich domain at the $\mathrm{C}$-terminus of $\mathrm{Gy}$ subunit. Rice varieties with the $D E P 1$ allele are characterized by the erect panicle architecture, well-developed vascular bundles, an increased number of grains per panicle and increased grain yield.

Huang et al. [14] investigated DEP1 homologues from T. urartu Thum. ex Gandil., T. aestivum L. and Hordeum vulgare $\mathrm{L}$., and identified variable deletions within 5 th exon in comparison with to the dominant allele described in rice. The detailed characterization of the HVDEP1 gene for the large collection of barley cultivars showed no correlation between $H v D E P 1$ gene sequences and ear phenotype [15]. The upstream open reading frame (uORF) identified in the $5^{\prime} \mathrm{UTR}$ of $H V D E P 1$ is predicted to contribute to post-transcriptional regulation of the gene. In case of $T$. aestivum the experiments with the transgenic line showed that downregulation of DEP1 homologue affects the length of the ear, ear density and number of spikelets [14]. Thus, the identification of this gene in wheat species with different spike shape will expand our knowledge of $D E P 1$ role in the evolution of cereals.

\section{Methods}

\section{Plant materials}

Germplasm of $T$. monococcum L. (4 accessions), $T$. durum Desf. (2 accessions), T. compactum (1 accession), and T. spelta (1 accession), were obtained from Gifu University (Gifu, Japan), Graduate School of Agriculture of Kyoto University (Kyoto, Japan), N.I. Vavilov AllRussian Institute of Plant Industry (St-Petersburg, Russia), The Federal Research Center Institute of Cytology and Genetics The Siberian Branch of the Russian Academy of Sciences (Novosibirsk, Russia), National Small Grains Collection (Aberdeen, USA) and Kh.Yu. Yusufbekov Pamir biological institute (Khorog, Republic of Tajikistan). Ten plants of each accession were growing in the greenhouse under the standard conditions. Spike shape was determined visually and confirmed by calculation of Flaksberger's formula [16]. The significance of differences between accessions with normal and compact (or compactoid) spikes was determined by the Student test. The results are presented in the Table 1 and Additional file 1: Table S1.

\section{DEP1 gene extraction from WGS databases}

Full-length sequences of DEP1 gene of $T$. aestivum, $T$. urartu, and Aegilops tauschii Coss. were extracted from Whole-Genome Shotgun contigs (WGS) database (https://www.ncbi.nlm.nih.gov/genbank/wgs/) applying Basic Local Alignment Search Tool (BLAST) (Additional file 1: Table S2) [17]. DEP1 mRNA sequences of T. aestivum (GenBank No. FJ039902) and T. urartu (GenBank No. GQ324995) were used as query sequences for the BLAST search.

\section{DEP1 gene cloning and sequencing}

Total DNA was isolated from $100 \mathrm{mg}$ of leaves using the DNeasy Plant Mini Kit (QIAGEN) according to the manufacturer's protocol.

The DEP1 mRNA sequences of T. urartu (GQ324995), T. aestivum (FJ039902) and H. vulgare (FJ039903) were used to design primers to amplify DEP1 gene from the $T$. monococcum, $T$. durum, $T$. compactum, and $T$. spelta. Primer pair for the amplification of 5 'UTR of the DEP1 was constructed using full-length $D E P 1$ sequence of $T$. aestivum extracted from WGS database (Additional file 1: Table S2). The genomic DEP1 gene sequence was PCR-amplified as five separate overlapping fragments using primers pairs listed in Additional file 1: Table S3. Schematic representation of the primer pairs positions and PCR conditions for each fragment are shown in the Additional file 1: Table S3 and Figure S1. PCR products were separated by agarose gel electrophoresis and purified using a QIAquick Gel Extraction Kit (QIAGEN). For T. monococcum accessions purified PCR products were sequenced directly without cloning. For polyploid wheat species purified PCR fragments were cloned into a pGEM ${ }^{\circ}-\mathrm{T}$ Easy vector using a pGEM-T Easy kit (Promega) and amplified with M13 primers prior to sequencing (Additional file 1: Table S3). Sequencing reactions were performed with $200 \mathrm{ng}$ of the PCR product and ABI BigDye Terminator Kit on an ABI 3130XL Genetic 
Table 1 List of wheat accessions used for sequence analysis of the DEP1 gene

\begin{tabular}{|c|c|c|c|c|c|}
\hline Genome & Species & Accession number & Place of origin & Spike shape & DEP1 GenBank No. \\
\hline$\overline{A^{b}}$ & T. monococcum L. & Sog glume-1 & Mutant obtained in Japan [24] & compactoid & MF979621 \\
\hline$A^{b}$ & T. monococcum L. & Sog glume-2 & Mutant obtained in Japan [24] & compactoid & MF979622 \\
\hline$A^{b}$ & T. monococcum L. & Extremely early $C$ & Mutant obtained in Japan & normal & MF979623 \\
\hline$A^{b}$ & T. monococcum L. & K-18105 & Nagorno-Karabakh & normal & MF979624 \\
\hline$B A^{u}$ & T. durum Desf. & Sharik & Russia [25] & compactoid & MF979625, MF979631 \\
\hline $\mathrm{BA}^{\mathrm{u}}$ & T. durum Desf. & $\begin{array}{l}\text { Lnd222 } \\
\text { (Cl12341) }\end{array}$ & USA & normal & MF979626, MF979632 \\
\hline$B A^{\mathrm{u}} \mathrm{D}$ & T. compactum Host & K1711 & Gorno-Badakhshan Autonomous Region & compact & $\begin{array}{l}\text { MF979627, MF979629 } \\
\text { MF979633 }\end{array}$ \\
\hline$B A^{\mathrm{u}} \mathrm{D}$ & T. spelta L. & K-53660 & Gorno-Badakhshan Autonomous Region & Normal & $\begin{array}{l}\text { MF979628, MF979630 } \\
\text { MF979634 }\end{array}$ \\
\hline
\end{tabular}

Analyser (Applied Biosystems) in SB RAS Genomics Core Facility (http://www.niboch.nsc.ru/doku.php/corefacility). In total 10 clones were sequenced for each $D E P 1$ fragment from 4 accessions of $T$. durum, T. spelta and T. compactum. DEP1 gene sequences were deposited in GenBank (Table 1).

\section{DEP1 gene sequences analyses}

Nucleotide and amino acid sequences alignments were performed using Vector NTI Advance version 10.0 program and improved with the MUSCLE algorithm in UGENE software (http://ugene.unipro.ru/) [18, 19]. ORFs in DNA sequences were predicted using ORF Finder program available from National Center for Biotechnology Information (NCBI) (https://www.ncbi.nlm.nih.gov/orffinder/). Phylogenetic analysis was performed by Maximum likelihood method (ML) integrated in PhyML 3.0 program [20]. Statistical support for the ML tree was evaluated by bootstrapping [21]. p-distances for DEP1 and VRN1 genes sequences were calculated using MEGA5 software [22]. UGENE software was used for the multiple sequence alignments visualization and editing [19].

\section{Results \\ Variability of the predicted 5th exon of DEP1 gene in wheat species}

In rice $D E P 1$ gene the gain-of-function mutation is located within the 5th exon region. Using the Dep1-MF/Dep1MR primer pair we obtained the sequences of the predicted 5th exon of the DEP1 gene for the Triticum species listed in the Table 1. A and D genome copies of DEP1 gene of T. aestivum (FAOM01374184 - 5AL chromosome, FAOM01435944 - 5DL chromosome) and DEP1 gene sequence of Ae. tauschii (MCGU01048243) were extracted from WGS database. The third DEP1 sequence of T. aestivum (CCYC011397742) extracted from WGS database is predicted to be $\mathrm{B}$ genome homologues of the gene. To investigate differences between the predicted 5th exon sequences, we aligned the newly obtained sequences and sequences extracted from WGS database with DEP1 mRNA of T. urartu (GQ324995), T. aestivum (FJ039902) and $H$. vulgare (FJ039903). The results of comparative analysis are presented in the Additional file 1: Figure S2.

One variant of the predicted 5th exon sequence $639 \mathrm{bp}$ in length was detected for the analyzed $T$. monococcum accessions. We observed one sequence change that distinguishes accessions with compactoid and normal spikes: $\mathrm{C} /$ $\mathrm{T}$ substitution in the position 375 didn't lead to amino acid substitution (Fig. 1a, Additional file 1: Figure S2).

Two different sequences of the predicted 5 th exon (639 bp and $579 \mathrm{bp}$ in length) were identified for the accessions of $T$. durum. Longer sequence of $T$. durum Lnd222 was identical to sequence of $T$. aestivum (FAOM01374184 - 5AL chromosome) (Fig. 1a). The second sequence from $T$. durum Lnd222 differed from A genome copy by five deletions and 24 nucleotide substitutions that caused 9 amino acid changes (Additional file 1: Figure S2). In case of T. durum Sharik the second sequence of the 5 th exon was separated by the same five deletions and 23 nucleotide substitutions (Additional file 1: Figure S2). Analysis of the ORFs showed that only 8 out of 23 substitutions led to the amino acid changes. Shorter sequences from $T$. durum Sharik and T. durum Lnd222 were similar to the DEP1 from T. aestivum (CCYC011397742) and are predicted to refer to $D E P 1-B$ gene (Fig. 1c, Additional file 1: Figure S2). Nucleotide sequences of the 5th exon of DEP1-A of $T$. durum accessions with normal and compactoid spikes differed by two substitutions (positions 213 and 242). Substitution CCT/CTT in codon 218 led to amino acid substitution P/L (Fig. 1a). No differences in DEP1-B sequences which separate $T$. durum accessions with normal and compactoid spikes were identified (Fig. 1c).

Three sequences of the predicted 5th exon were obtained for $T$. compactum $\mathrm{K} 1711$ and $T$. spelta K53660. Sequences of $639 \mathrm{bp}$ in length were identical for these accessions and showed $99.8 \%$ similarity with DEP1 sequence of $T$. aestivum (FAOM01374184 - 5AL 


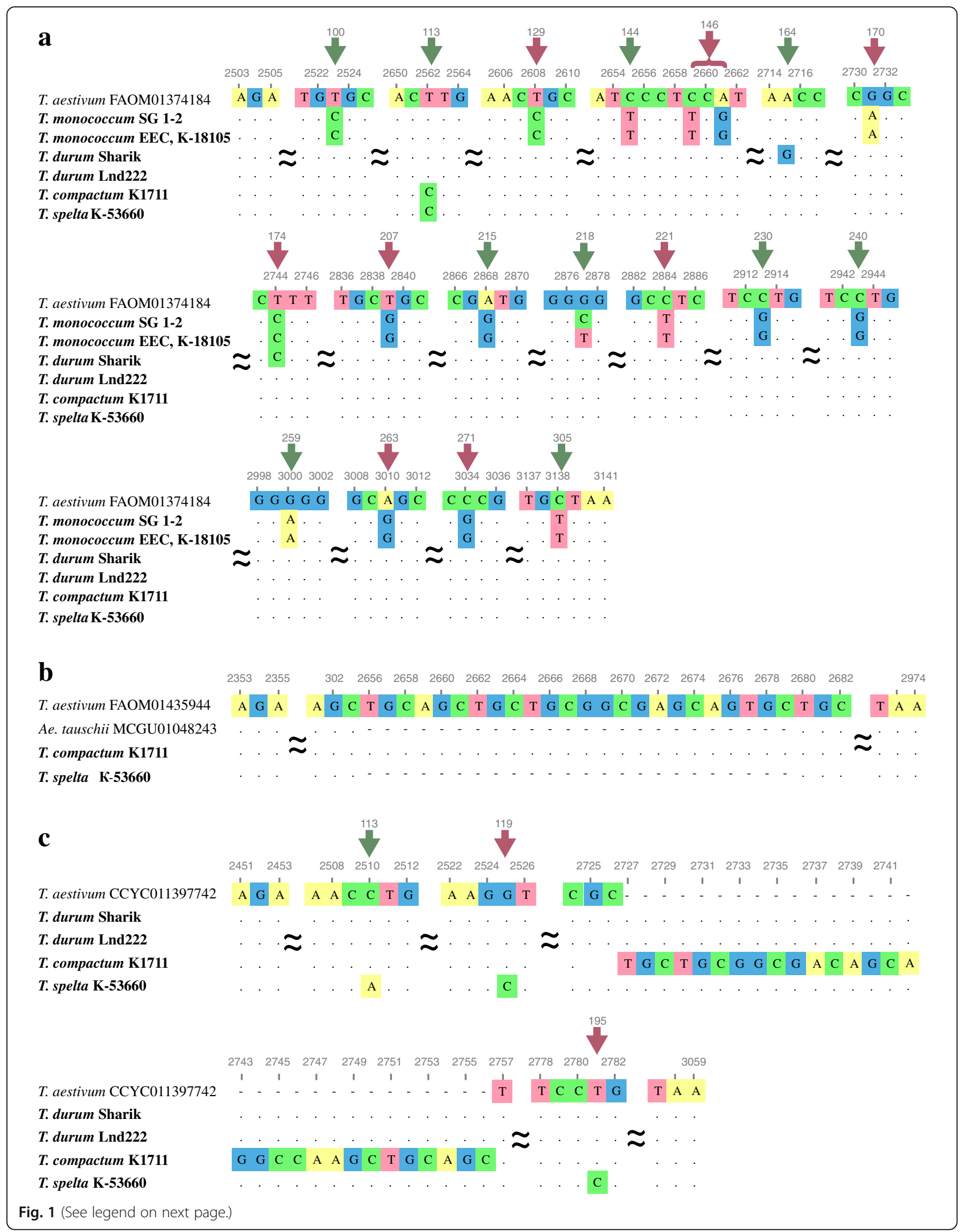


(See figure on previous page.)

Fig. 1 Variability of 5th exon of DEP1 gene from di-, tetra- and hexaploid wheat species. a Newly obtained DEP1-A sequences from T. monococcum, T. durum, T. compactum, T. spelta and reference sequence of T. aestivum (FAOM01374184) extracted from WGS database. b Newly obtained DEP1-D sequences of $T$. compactum and T. spelta and reference sequence of $T$. aestivum (FAOM01435944) extracted from WGS database. c Predicted B genome homologues of DEP1 gene from T. durum, T. compactum, T. spelta and reference sequence T. aestivum (CCYC011397742) extracted from WGS database. Wheat accessions analyzed in the present study are marked in bold. Nucleotides matching with reference sequence are designated by dots. Nonsynonymous and synonymous amino acid substitutions are indicated by red and green arrows, respectively. Numbers of the nucleotides upstream from the start codon are given in accordance with the DEP1 sequences of $T$. aestivum. Numbers of codons are presented above the rows according to DEP1 of T. aestivum

chromosome) (Fig. 1a, Additional file 1: Figure S2). D genome copies of DEP1 the predicted 5th exon from analyzed T. compactum $\mathrm{K} 1711$ and T. spelta K-53660 accessions showed 100 and $96 \%$ similarity to T. aestivum FAOM01435944, correspondingly. T. spelta accession possess a $24 \mathrm{bp}$ deletion from position 304 to 328 when comparing to $T$. compactum $\mathrm{K} 1711$ and $T$. aestivum FAOM01435944 (Fig. 1b, Additional file 1: Figure S2). Analogous deletion is presented in Ae. tauschii (MCGU01048243). The deletions described above did not cause frame shifts as it was shown for the recessive allele of $D E P 1$ gene in O. sativa [14]. The third variants of the predicted 5 th exon sequences of $T$. compactum K1711 differed by one insertion of $30 \mathrm{bp}$ in length and showed high similarity to the sequences of $T$. aestivum (CCYC011397742). The last variant of the predicted 5th exon sequences of T. spelta K-53660 showed $99.5 \%$ similarity with DEP1 sequence of $T$. aestivum (CCYC011397742) (Fig. 1c, Additional file 1: Figure S2).

\section{Variability of 5'UTR of DEP1 gene in wheat species}

We identified 5' UTR (approximate length 250 bp) of DEP1 gene for accessions of T. monococcum, T. durum, T. compactum и T. spelta using Dep1-LF/Dep1-LR primer pair (Additional file 1: Table S1). One variant of 5' UTR was obtained for each accession of analyzed diand tetraploid wheat species. Length of the sequence was $254 \mathrm{bp}$ and $256 \mathrm{bp}$ in T. monococcum and T. durum, correspondently. For both T. compactum $\mathrm{K} 1711$ and $T$. spelta K-53660 we obtained two variants of 5'UTR of $256 \mathrm{bp}$ and $259 \mathrm{bp}$ in length.

ORFs were predicted for newly obtained sequences and 5'UTRs of DEP1 from wheat, barley and Aegilops available from databases. The results showed that all 5'UTRs from species listed in Table 1 possess the uORF that varied in length from 63 to 98 amino acids (Fig. 2). N-terminus of the predicted amino acid sequences was conservative among studied species while the rest part varied highly. uORFs of $T$. aestivum (FAOM01374184), accessions of T. durum and

\begin{tabular}{|c|c|c|}
\hline DEPl uORF Hv* & 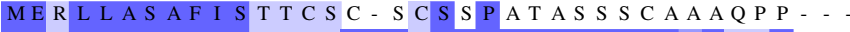 & $--\mathrm{H}$ \\
\hline DEPl uORF Ta (FAOM01374184) & E G L L A S A F I S T T C S A L L R S A P P Q P T P P P P S P R V A - & - \\
\hline DEP1 uORF Tm SG 1-2, EEC, K-18105 & I G L L A S A F I S T T C S A L L R S A P P Q P T P P P P S P R V A - & - \\
\hline DEP1 & MEGLLASAF I ST TCS A L L R A P P Q P T P P P P S P R A - & - \\
\hline$D E P 1$ & MEGLLASAF I ST T C S A L LR S A P P P T P P P P S P R V A - & - $R$ \\
\hline$D E$ & E G L L A S A F I S T T C S A L L R S A P P Q P T P P P P S P R VA. & $-\ldots-\ldots-\ldots$ \\
\hline$D E P I$ & MER L L A S A F I S L P P P A L L C S A P P Q P T P P P P S P R V A - & - - R T T H A R T H - \\
\hline$D E P$ & MER L L A S A F I S L P P P A L L C S & - - R - T H A R T H - L \\
\hline$D E$ & IERLLASAFISFP P A L LCSAP P P T P P P P P P P A S I & S S H R - T H A R T H A L \\
\hline$D E$ & MER L L A S A I S F P P P A L C S A P Q P T P P P P P S P A S I & S S HR - THAR THAL \\
\hline & MER L L A S A F I S F P P P A L L C A P P Q P T P P P P P P P A S I S & S S H R - THAR THA L \\
\hline$D E P$ & MERLLASAF I S F P P P A L LCSA P P P T P P P P S P A I - - S & S S H R - T H A R T H A \\
\hline$D E P$ & S T I T A A Q Y A T S T P S F A $\ldots$. & - - C C C C C S S G G D \\
\hline$D E P$ & R S P A P L R F L A . . . . . . . . . S P P A S A Q P & P L P R A S C C - - - \\
\hline$D E P$ & R S P A P L R F L A ............. S P P A S A Q P & P L P R A S Y R - - \\
\hline$D E P$ & R S P A P L R F L A . . . . & P L P R A S C C - - - \\
\hline$D E P$ & $\ldots \ldots-\ldots$ P P A S A Q P I & P L P R A S C C - - - \\
\hline$D E P$ & R S P A P L R F L A $\ldots \ldots \ldots$ S P A S A Q P & P L P R A S C C - - - \\
\hline$D E P$ & C S D S L L H H R P VR NHCS T . . . . . . AP P A A S . & $\cdots-\cdots$ C R P A A G A \\
\hline$D E P$ & C S D S L L H H R P VR NHCST . . . . . . AP P A A S . & $\ldots-\ldots$ C P A A G A \\
\hline$D E P$ & C A A Q I P Y Y I TA - G Q C A T T A P R L L L L A A P P P A N AC & GR W L S L S P P G P G V \\
\hline$D E P$ & CA A I P Y Y I TA- G Q C ATTAP P R L L L A A A P P P A & G R W L S L S P P G P G V \\
\hline & Q I P Y Y I T A - G Q C A T T A P P R L L L L A A A P P P A & GR W L S L S P P G P G \\
\hline DEPl uORF Aet (MCGU01048243) & C A A I P Y Y I TA - G Q C A T TA P P R L L L A A A P P P P N AC & GR W L S L S P P G P G \\
\hline
\end{tabular}

Fig. 2 Alignment of upstream open reading frames, determined in 5'UTR of DEP1 gene from several grasses. Single asterisk indicates sequences identified by Belanger et al. [15]. Abbreviations: uORF - upstream open reading frame, Hv - Hordeum vulgare, Ta - Triticum aestivum, Tm - Triticum monococcum, Td - Triticum durum, Tc - Triticum compactum, Ts - Triticum spelta, Aet - Aegilops tauschii. Wheat accessions analyzed in the present study are marked in bold. Amino acid residues are colored according to the percentage identity in the UGENE software [19] 
first variant of $T$. compactum $\mathrm{K} 1711$ and $T$. spelta $\mathrm{K}$ 53660 uORFs were $63 \mathrm{bp}$ in length and identical to each other. Sequences from studied T. monococcum accessions differed from listed above by two amino acid changes. Length of uORF predicted for $T$. aestivum (CCYC011397742) was 76 amino acids. The second variant of uORF from T. aestivum (FAOM01435944), T. compactum K1711 and T. spelta K-53660 were identical (length 98 amino acids) and differed from the uORF of Ae. tauschii (MCGU01048243) by to amino acid substitution (Fig. 2).

uORFs were identified for DEP1 genes of all studied accessions and thus confirm the suggestion that presence of uORF is conservative characteristic of orthologous genes DEP1 in grasses [15].

\section{Full-length DEP1 gene sequence: comparative and phylogenetic analysis}

To investigate variability within predicted introns and the first 4 exons of the DEP1 gene we obtained fulllength gene sequences from 8 accessions of di-, tetra, and polyploid wheat species (Table 1). Four DEP1 nucleotide sequences of $T$. monococcum accessions were identified by direct sequencing of PCR fragments. In case of polyploid species were identified DEP1 sequences by PCR, cloning and sequencing. Full-length sequences were constructed by alignment of overlapping fragments.

\section{Comparative analysis of DEP1 gene from A genome}

Figure 3 illustrates variable and conservative regions of $D E P 1$ gene found in the wheat species studied and $A e$. tauschii. Nucleotide sequences of all four accessions of $T$. monococcum encode identical protein sequences, although two of those accessions have compactoid spikes and the other two have normal spike. The only difference between $D E P 1$ gene sequences from accessions with compactoid and normal spikes is the length of the (TC) track within the first intron. Nucleotide sequences of T. monococcum Sog glume-1 and T. monococcum Sog glume-2 have (TC) repeat, while accessions Extremely early C and K-18105 have (TC) ${ }_{11}$ repeat (Fig. 3a).

Several DEP1 sequences were obtained for polyploid wheat accessions. In each accession of $T$. durum, T. compactum and $T$. spelta we identified DEP1 sequence that was identical to $T$. aestivum (FAOM01374184 - 5AL chromosome) in the coding region and thus, was referred to the A genome. However, non-coding regions of DEP1-A are characterized by minor differences (Fig. 3b). 5' UTR of T. spelta K-53660 possesses a deletion of $12 \mathrm{bp}$, while $T$. urartu possesses a deletion of two nucleotides in the first intron and an insertion of two nucleotides in the fourth intron. In addition, nucleotide sequence of the intron 2 of $T$. urartu contains five nucleotide substitutions compared to the sequences of DEP1 gene of other accessions. Finally, the nucleotide sequences of T. urartu and T. durum Sharik accessions differ from sequences of $T$. aestivum (FAOM01374184), T. durum Lnd222, T. spelta K-53660 and T. compactum K1711 accessions by six nucleotide

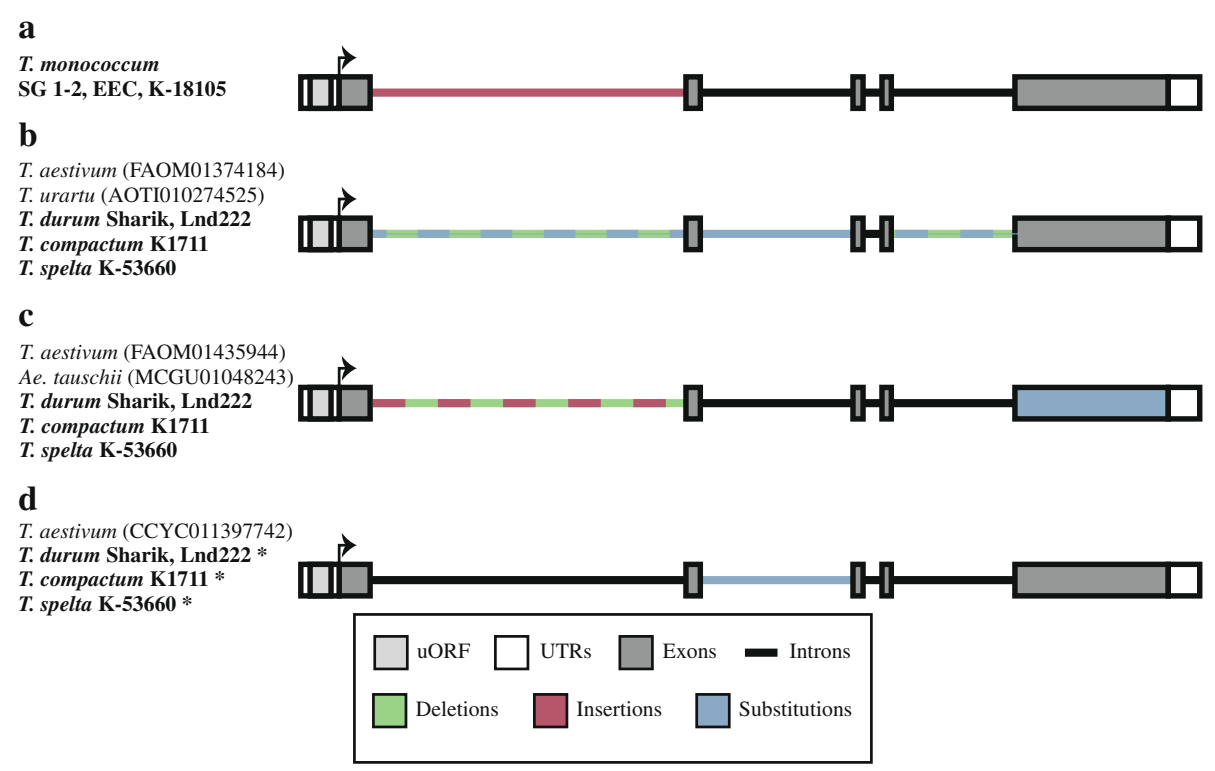

Fig. 3 Scheme of variable and conservative regions of DEP1 gene from wheat species and Aegilops tauschii. a DEP1 gene from Triticum monococcum accessions. b DEP1-A gene from polyploid wheat species and diploid Triticum urartu. c DEP1-D gene from polyploid wheat species and Aegilops tauschii. $\mathbf{d}$ Predicted B genome homologues of DEP1 gene from wheat species. Wheat accessions analyzed in the present study are marked in bold 
substitutions in the first intron and one nucleotide substitution in the fourth intron (Fig. 3b).

\section{Comparative analysis of DEP1 gene from $D$ genome}

In the $T$. compactum $\mathrm{K} 1711$ and $T$. spelta K-53660 we identified DEP1 sequences similar to the $T$. aestivum (FAOM01435944 - 5DL chromosome) and Ae. tauschii (MCGU01048243) and, thus as a result were referred to the $\mathrm{D}$ genome. In contrast to $\mathrm{A}$ genome, the $D E P 1$ gene sequences of D genome showed variability in coding region among studied species (Fig. 3c). Ae. tauschii (MCGU01048243) and T. spelta K-53660 contain a deletion of 24 nucleotides in compare to $T$. aestivum (FAOM01435944) and T. compactum K1711. Differences are presented in the non-coding regions as well. The DEP1 gene of Ae. tauschii possesses a deletion of six nucleotides in the 5' UTR and two insertions in the first intron of 1 and 5 bp in length. 5' UTR and the first intron of T. spelta K-53660 contain deletions and insertions, however, their positions are different from Ae. tauschii (Fig. 3c).

\section{Comparative analysis of predicted $B$ genome homologues of DEP1 gene}

Additional variant of $D E P 1$ identified for the accessions of polyploid wheat showed high similarity to DEP1 of T. aestivum (CCYC011397742) (Fig. 3d). These sequences were obtained partially and 5'UTR, exon 1 and intron 1 remain unknown. Comparative analysis of all these sequences showed no differences in the coding region, although several nucleotide substitutions were found in the predicted intron 2 (Fig. 3d). In comparison with T. aestivum, T. spelta K-53660 had three nucleotide substitutions, while T. compactum K1711 had two nucleotide substitutions, and both samples of $T$. durum had no substitutions at all. These sequences were predicted to be the $D E P 1-B$ gene and showed 8.2 and $4.6 \%$ dissimilarity to the DEP1- $A$ and $D E P 1-D$ sequences, correspondingly. However, within genomes p-distances were less and varied 0-3.4 and 0.2$0.3 \%$ in case of $D E P 1-A$ and $D E P 1-D$ gene sequences, correspondingly (Additional file 1: Table S4). Similar situation was detected for another wheat gene VRN1. Distance between VRN1 alleles from the same genome was lower than distance between sequences from different genomes (Additional file 1: Table S5).

\section{Phylogenetic analysis of DEP1 genes}

Phylogenetic analysis was performed using DNA sequences of newly obtained DEP1 genes sequences from different wheat species (Table 1). Sequence of the DEP1 from O. sativa (FJ039904) was used as an outgroup. Four clusters in addition to the outgroup are presented in the phylogenetic tree (Fig. 4). The first cluster (I) is formed by the DEP1 gene sequences from $\mathrm{A}^{\mathrm{b}}$ genome of T. monococcum accessions. DEP1 sequences of these accessions are grouped into two clades according to the spike shape (compactoid or normal). The second cluster (II) consists of DEP1 sequences from five species: $T$. urartu, T. aestivum, T. durum, T. spelta and T. compactum (Fig 4). These sequences belong to the $\mathrm{A}^{\mathrm{u}}$ genome and are grouped together but not according to spike shape of wheat accessions. All DEP1 sequences from D genomes of wheat species are in the III cluster together with DEP1 of Ae. tauschii. The last cluster (IV) is

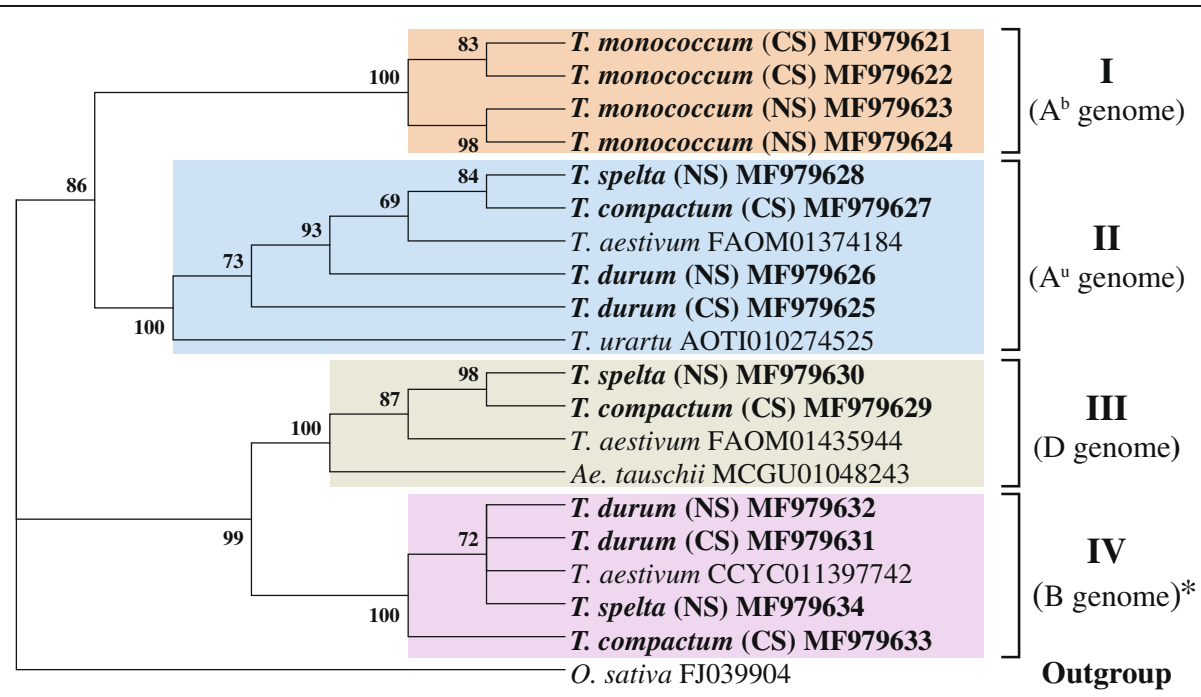

Fig. 4 Maximum likelihood phylogenetic tree constructed based on DEP1 gene sequences from wheat species and Aegilops. OsDEP1 gene sequence from O. sativa (FJ039904) was used as outgroup. Statistical support was evaluated by bootstrapping (1000 replications); nodes with bootstrap values over $50 \%$ are indicated. Clusters are highlighted in different colors. Sequences obtained in this study are marked in bold. Abbreviations: CS - compact or compactoid spike, NS - normal spike. * - predicted B genome 
formed by the $D E P 1$ sequences which are highly similar to the predicted $\mathrm{B}$ genome homologues of DEP1 of $T$. aestivum (CCYC011397742). The DEP1 sequences from polyploid wheats were not grouped in the phylogenetic tree according to the spike phenotype (Fig. 4).

\section{Discussion}

\section{DEP1 gene and spike shape trait in wheat}

Genetic control of spike shape, which is one of the main morphological trait of the wheat species, is poorly known. Nowadays only $Q$ gene which pleiotropically affect spike shape trait in polyploid wheat species was well studied [9-11]. Other loci related to the trait such as $C, C 2, s c 1$ and $s c 2$ are poorly investigated $[3,5,8]$. We choose $D E P 1$ gene previously identified in rice as a candidate for the role of spike shape trait regulator in wheat. In rice the $D E P 1$ is a dominant negative regulator of panicle architecture and grain number [14]. Homologues of the gene were characterized in $5 \mathrm{H}$ chromosome of barley [15]. In case of wheat species only mRNAs of DEP1 were identified for T. aestivum и $T$. urartu. The above-mentioned species are characterized by normal spike shape and did not allow to investigate the influence of $D E P 1$ gene on spike shape trait.

In this study full-length gene sequence of $D E P 1$ was identified for the eight accessions of four wheat species icluding di-, tetra- and hexaploids with normal, compact and compactoid spikes (Table 1). Furthermore, fulllength DEP1 sequences of T. aestivum, T. urartu and Ae. tauschii were extracted from the WGS database. Comparative and phylogenetic analysis of obtained DEP1 sequences revealed no mutations that distinguish accessions with normal spikes from compact or compactoid phenotype (Figs. 1, 2 and 3). Moreover, in case of $T$. monососсит и $T$. durum no intraspecific variability of the coding region of $D E P 1$ gene was identified despite investigation of both normal and compactoid accessions of the same species. Therefore, the results of the study rose the questions about the influence of the DEP1 gene on spike architecture in wheat.

Belanger et al. [15] performed similar analysis for the $D E P 1$ in various two-row and six-row accessions of barley from different geographic origins. They also did not identify the gene sequence variability which could separate accessions with different phenotype. All identified mutations were located within introns except for three SNPs in the first and the fifth exon that however resulted in silent mutations [15]. However, relatively long 5' UTR of DEP1 encoding peptide of 70 amino acids was identified and predicted to contribute to post-transcriptional regulation of the gene. We identified uORFs within the 5' UTR of DEP1 genes for all studied species and suggested that it could contribute to the post-transcriptional regulation of $D E P 1$ and formation of normal or compact (compactoid) spikes in wheat (Fig. 2).

Although we did not identify mutation within DEP1 gene sequence, which could distinguish wheat accessions based on the spike morphology. This gene takes part in the regulation of the spike shape trait. Huang et al. [14] showed that downregulation of DEP1 in $T$. aestivum leads to increased length of the ear, makes it less compact and reduces number of spikelet. Thus, investigation of other loci related to the spike shape trait is essential to determine mode of the $D E P 1$ influence.

\section{DEP1 gene and origin of Triticum species}

Previously, we suggested the scheme of the wheat species origin and evolution [23]. The results of this study supported the above-mentioned scheme. The $D E P 1$ gene from $\mathrm{A}^{\mathrm{b}}$ genome was presented only in T. monococcum accessions and was not identified for the polyploid wheat species. This fact supported the hypothesis that $\mathrm{A}^{\mathrm{b}}$ genome of the T. monococcum was inherited from T. boeoticum and was not presented among polyploid wheat species. T. urartu is a donor of second variant of the A genome $\left(\mathrm{A}^{\mathrm{u}}\right)$ for all polyploid wheats. In this study one variant of $D E P 1$ gene was identified for both $T$. urartu and polyploids analyzed. On the phylogenetic tree $D E P 1$ gene from $T$. urartu took basal position towards $T$. durum, T. aestivum, T. compactum and T. spelta. Thus, we supported the hypothesis of the inheritance of $\mathrm{A}^{\mathrm{u}}$ genome by polyploid wheat species from $T$. urartu.

According to the scheme [23] the Ae. tauschii is a donor of D genome for hexaploid wheat species of section Emmer. Thus, this study supports this way of inheritance. Indeed, the variant of DEP1 gene extracted from WGS database was identified in hexaploid wheat species and absent in tetraploid species T. durum (Figs. 3 and 4). B genome of tetra- and hexaploid wheat species originated from Ae. speltoides. The sequence of DEP1 gene from Ae. speltoides is unknown, but one of the gene variant identified among polyploids wheat only is predicted to be a $\mathrm{B}$ genome homologue of this gene. The level of dissimilarity between predicted $D E P 1-B$ sequences and $D E P 1-A$ and $D E P 1-D$ sequences is higher than dissimilarity within sequences from the same genome. The same situation was shown for another wheat gene VRN1 (Additional file 1: Table S4). On the phylogenetic tree predicted $D E P 1-B$ sequences were grouped into one cluster separated from DEP1-A and DEP1-D clusters (Fig. 4). Thus, the results of comparative and phylogenetic analysis showed that the predicted $D E P 1-B$ sequences are not the new alleles of $\mathrm{A}$ or $\mathrm{D}$ genomes and support the suggestion that DEP1 of T. aestivum (CCYC011397742) and sequences of $T$. durum (MF979631, MF979632), T. spelta (MF979634) and T. 
compactum (MF979633) represent B genome homologues of DEP1.

\section{Conclusions}

In this study full-length $D E P 1$ gene sequences from 8 accessions of di-, tetra-, and hexaploid wheat species were obtained. In addition, full-length sequences of DEP1 gene of T. aestivum, T. urartu and Aegilops tauschii were extracted from WGS database. The comparative and phylogenetic analysis revealed that tetraploid wheat $T$. durum contained two variants of the DEP1 belonged to $\mathrm{A}$ and $\mathrm{B}$ genomes. Three variants of this gene belonged to $\mathrm{A}, \mathrm{B}$, and $\mathrm{D}$ genomes have been found in the hexaploid wheats $T$. aestivum, T. compactum and T. spelta. $D E P 1$ genes of the diploid wheats T. monococcum and $T$. urartu were different. It means that a precursor of the DEP1 gene in T. monococcum should be from T. boeoticum. All the sequences obtained were species specific. DEP1 gene mutations, distinguishing wheat accessions based on the spike morphology, were not found. uORFs were identified within the 5' UTR of DEP1 genes for all studied accessions. The uORF could contribute to the post-transcriptional regulation of $D E P 1$ gene and formation of normal or compact (compactoid) spikes in wheat. Thus, further investigations are required to determine $D E P 1$ gene engagement in the regulation of the spike shape trait.

\section{Additional file}

Additional file 1: Table S1. Morphological characteristics of the wheat species used in the present study. Table S2. List of DEP1 gene sequences of Triticum and Aegilops species obtained from WGS database. Table S3. Set of primers and PCR conditions used in the present study. Table S4. p-distances between wheat DEP1 genes sequences from A, B and D genomes. Table S5. $p$-distances between wheat VRN1 genes sequences from A, B and D genomes. Figure S1. Schematic representation of the primer pairs positions. Figure S2. Nucleotide alignment of 5th exon of DEP1 gene from different wheat species, Ae. tauschii and barley. Sequence of $T$. aestivum (FAOM01374184) was used as a reference. Sequences obtained by experimental methods in the present study are marked in bold. Nucleotides, that match with reference sequence, are designated by dots. Nonsynonymous and synonymous amino acid substitutions are indicated by red and green arrows, respectively. (PDF 8916 kb)

\section{Acknowledgments}

The authors are grateful to E.Ya. Kondratenko for technical support. The authors thank the reviewers for valuable discussion of the results.

\section{Funding}

Funding for this work was provided by the Russian Science Foundation (grant number: 16-16-10021). Publication of this article has been funded by the Russian Science Foundation (grant number: 16-16-10021).

\section{Availability of data and materials}

The DEP1 gene sequences are available in GenBank, with the accession numbers MF979621- MF979634.

\section{About this supplement}

This article has been published as part of BMC Genetics Volume 18 Supplement 1, 2017: Selected articles from Belyaev Conference 2017: genetics. The full contents of the supplement are available online at https://bmcgenet.biomedcentral.com/articles/supplements/volume-18supplement-1.

\section{Authors' contributions}

W cloned and sequenced DEP1 gene and contributed to the preparation of the manuscript. IK designed the molecular genetics experiments and wrote the manuscript. AK analyzed the results of the greenhouse experiments. $A B$ analyzed the results of molecular genetics experiments and participated in preparing the manuscript. NPG designed the greenhouse experiments and revised the manuscript. All the authors read and approved the final manuscript.

Ethics approval and consent to participate

Not applicable.

\section{Consent for publication}

Not applicable.

\section{Competing interests}

The authors declare that they have no competing interests.

\section{Publisher's Note}

Springer Nature remains neutral with regard to jurisdictional claims in published maps and institutional affiliations.

Published: 28 December 2017

\section{References}

1. Dorofeev VF, Korovina ON, editors. Kul'turnaya flora SSSR: Pshenitsa (Cultivated Flora of the Soviet Union: Wheat). Leningrad, Kolos, Leningrad branch; 1979 .

2. Malinowski E. Les hybrides du froment. Bull Acad Sci Cracovie. 1914;3:410-50.

3. Goncharov NP. Comparative genetic study of tetraploid forms of common wheat without D genome. Genetika. 1997;33:660-63.

4. Konopatskaia I, Vavilova V, Blinov A, Goncharov NP. Spike morphology genes in wheat species (Triticum L.). Proc Latv Acad Sci Sect B. 2016;70:345-55.

5. Rao MVP. Mapping of the compactum gene $C$ on chromosome $2 \mathrm{D}$ of wheat. Wheat Inf Serv. 1972;35:9.

6. Faris JD, Gill BS. Genomic targeting and high-resolution mapping of the domestication gene. Genome. 2002;45:706-18.

7. Faris JD, Fellers JP, Brooks SA, Gill BS. A bacterial artificial chromosome contig spanning the major domestication locus Q in wheat and identification of a candidate gene. Genetics. 2003;164:311-21.

8. Goncharov NP, Gaidalenok RF. Localization of genes controlling spherical grain and compact ear in Triticum antiquorum Heer ex Udacz. Russ J Genet. 2005:41:1262-7.

9. Simons KJ, Fellers JP, Trick HN, Zhang Z, Tai YS, Gill BS, et al. Molecular characterization of the major wheat domestication gene Q. Genetics. 2006; 172:547-55.

10. Zhang Z, Belcram H, Magdelenat G, Couloux A, Samain S, Gill BS, et al, Duplication and partitioning in evolution and function of homoeologous $Q$ loci governing domestication characters in polyploid wheat. Proc Natl Acad Sci U S A. 2011;108:18737-42.

11. Sormacheva I, Golovnina K, Vavilova V, Kosuge K, Watanabe N, Blinov A, et al. $Q$ gene variability in wheat species with different spike morphology. Genet Resour Crop Evol. 2015;62:837-52.

12. $\mathrm{Xu} \mathrm{H}$, Zhao $\mathrm{M}$, Zhang $\mathrm{Q}, \mathrm{Xu} \mathrm{Z}, \mathrm{Xu} \mathrm{Q}$. The DENSE AND ERECT PANICLE 1 (DEP1) gene offering the potential in the breeding of high-yielding rice. Breed Sci. 2016;66:659-67.

13. Xu Q, Zhao M, Wu K, Fu X, Liu Q. Emerging insights into heterotrimeric G protein signaling in plants. J Genet Genomics. 2016;43:495-502.

14. Huang X, Qian Q, Liu Z, Sun H, He S, Luo D, et al. Natural variation at the DEP1 locus enhances grain yield in rice. Nat Genet. 2009:41:494-7.

15. Bélanger S, Gauthier M, Jean M, Sato K, Belzile F. Genomic characterization of the Hordeum vulgare DEP1 (HVDEP1) gene and its diversity in a collection of barley accessions. Euphytica. 2014;1:29-41. 
16. Flaksberger KA. Pshenitsi- rod Triticum L. (Wheats- genus Triticum L.). Kul'turnaya flora SSSR. Khlebnie zlaki. Pshenitsa. (Cultivated Flora Sov. Union. Cereal. Wheat.). Gosudarstvennoe izdatel'stvo sovhoznoi i kolhoznoi literaturi. Moskva- Leningrad; 1935. p. 17-434.

17. Altschul SF, Gish W, Miller W, Myers EW, Lipman DJ. Basic local alignment search tool. J Mol Biol. 1990;215:403-10.

18. Lu G, Moriyama EN. Vector NTI, a balanced all-in-one sequence analysis suite. Brief Bioinform. 2004;5:378-88.

19. Okonechnikov K, Golosova O, Fursov M, Varlamov A, Vaskin Y, Efremov I, et al. Unipro UGENE: a unified bioinformatics toolkit. Bioinformatics. 2012;28:1166-7.

20. Guindon S, Dufayard J-F, Lefort V, Anisimova M, Horijk W, Gascuel O. New algorithms and methods to estimate maximum-likelihood phylogenies: assessing the performance of PhyML 3.0. Syst Biol. 2010;59:307-21.

21. Felsenstein J. Phylogenies and the comparative method. Am Nat. 1985;125:1-15.

22. Tamura K, Peterson D, Peterson N, Stecher G, Nei M, Kumar S. MEGA5:

Molecular evolutionary genetics analysis using maximum likelihood, evolutionary distance, and maximum parsimony methods. Mol Biol Evol. 2011;28:2731-9.

23. Goncharov NP, Golovnina KA, Kilian B, Glushkov S, Blinov A, Shumny VK. Evolutionary history of wheats - the main cereal of mankind. In: Dobretsov N, et al., editors. Biosph. Orig. Evol; 2008. p. 407-19.

24. Amagai Y, Burdenyuk-Tarasevych LA, Goncharov NP, Watanabe N. Microsatellite mapping of the loci for false glume and semi-compact spike in Triticum L. Genet. Resour. Crop Evol. Netherlands: Springer; 2017;1-9.

25. Goncharov NP, Mitina RL, Anfilova NA. Inheritance of awnlessness in tetraploid wheat species. Russ J Genet. 2003:39:463-6.

\section{Submit your next manuscript to BioMed Central and we will help you at every step:}

- We accept pre-submission inquiries

- Our selector tool helps you to find the most relevant journal

- We provide round the clock customer support

- Convenient online submission

- Thorough peer review

- Inclusion in PubMed and all major indexing services

- Maximum visibility for your research

Submit your manuscript at www.biomedcentral.com/submit 